\title{
Polynomials dividing infinitely many quadrinomials or quintinomials
}

\author{
by \\ L. Hajdu (Debrecen) and R. TiJdeman (Leiden)
}

1. Introduction. In this paper we give necessary and sufficient conditions for polynomials in $\mathbb{Q}[x]$ having only simple roots to divide infinitely many standard quadrinomials or quintinomials over $\mathbb{Q}$.

Let $K$ be any field of characteristic zero. A polynomial $P \in K[x]$ which is of the form

$$
P(x)=\sum_{i=1}^{k} a_{i} x^{m_{i}} \quad \text { with } m_{1}>\ldots>m_{k-1}>m_{k}=0 \text { and } a_{1}=1
$$

is called a standard $k$-nomial. For a standard $k$-nomial $P$ which is not a standard $(k-1)$-nomial, we call $\left(m_{1}, \ldots, m_{k}\right)$ the exponent $k$-tuple of $P$. Put

$$
\begin{array}{r}
\mathrm{PR}_{k}=\{P \in \mathbb{Q}[x]: \exists Q \in \mathbb{Q}[x] \text { and } r \in \mathbb{Z} \text { with } \operatorname{deg}(Q)<k \\
\text { and } \left.r \geq 1 \text { such that } P(x) \mid Q\left(x^{r}\right) \text { over } \mathbb{Q}\right\} .
\end{array}
$$

Two algebraic numbers $\beta_{1}, \beta_{2}$ are called equivalent if for some root of unity $\varepsilon$ we have $\beta_{1} \varepsilon=\beta_{2}$. For a polynomial $P \in \mathbb{Q}[x]$ having only simple roots we have $P \in \mathrm{PR}_{k}$ if and only if the roots of $P$ belong to the union of at most $k-1$ equivalence classes (cf. [6, Proposition 2.1]). We will use this assertion throughout the paper without any further reference.

In 1965 Posner and Rumsey noted (see [4, pp. 339 and 348]) that $P \in$ $\mathrm{PR}_{k}$ implies that $P$ divides infinitely many standard $k$-nomials over $\mathbb{Q}$. They proposed the converse as a conjecture, that is, if a polynomial $P \in \mathbb{Q}[x]$ divides infinitely many standard $k$-nomials over $\mathbb{Q}$, then $P \in \mathrm{PR}_{k}$. For $k=2$ the conjecture is obvious.

2000 Mathematics Subject Classification: Primary 11C08; Secondary 11D57.

The research was supported in part by the Netherlands Organization for Scientific Research (NWO). The first author was further supported by the Hungarian Academy of Sciences, by the János Bolyai Research Fellowship, by grants F034981 and T029330 of the Hungarian National Foundation for Scientific Research and by the FKFP grant $3272-13 / 066 / 2001$. 
At this point we mention that the restriction to standard $k$-nomials is only for convenience. We may replace every standard $k$-nomial by some constant multiple of it and the theorems would still be valid.

In [2] Györy and Schinzel proved the conjecture in a quantitative form for $k=3$. They gave an explicit expression $C_{1}$ such that if $P$ divides more than $C_{1}$ standard trinomials over $\mathbb{Q}$, then $P \in \mathrm{PR}_{3}$. Here $C_{1}$ is a number depending on the degree of $P$ and some other parameters. Later, Schlickewei and Viola [5] presented a value of $C_{1}$ which depends only on the degree of $P$.

On the other hand, Györy and Schinzel [2] disproved the conjecture for every $k \geq 4$ by giving counterexamples. For every $k \geq 2$ they provided a polynomial $P \in \mathbb{Q}[x]$ that divides infinitely many standard quadrinomials over $\mathbb{Q}$, but $P \notin \mathrm{PR}_{k}$. In fact the quadrinomials have a zero constant term and have therefore only three non-zero terms. The problem is more difficult for polynomials with non-zero constant terms. For every $k \geq 2$ Győry and Schinzel [2] also gave a $P \notin \mathrm{PR}_{k}$ which divides infinitely many standard quintinomials over $\mathbb{Q}$ with non-zero constant terms. They proposed the following problem instead of the disproved conjecture of Posner and Rumsey.

Let $k$ be an integer with $k \geq 4$. Is it true that a polynomial $P \in \mathbb{Q}[x]$ with $P(0) \neq 0$ divides infinitely many standard $k$-nomials with non-zero constant terms if and only if either $P \in \mathrm{PR}_{k}$, or $P$ divides a standard $[(k+1) / 2]$-nomial?

For $k \geq 6$ Hajdu [3] gave a negative answer to this question, providing other kinds of counterexamples. He proposed to modify the problem of Győry and Schinzel as follows.

Let $k$ be an integer with $k \geq 4$. Is it true that a polynomial $P \in \mathbb{Q}[x]$ with $P(0) \neq 0$ divides infinitely many standard $k$-nomials with non-zero constant terms if and only if either $P \in \mathrm{PR}_{k}$, or $P$ divides a standard $(k-2)$-nomial which divides infinitely many standard $k$-nomials over $\mathbb{Q}$ ?

Recently Schlickewei and Viola [6] described a so-called "proper" family $\mathcal{F}_{k}$ of standard $k$-nomials such that if a polynomial $P$ having only simple roots divides more than $C_{2}(k)$ elements of $\mathcal{F}_{k}$, then $P \in \mathrm{PR}_{k}$ (cf. Lemma 2).

In this paper we give necessary and sufficient conditions for a polynomial $P \in \mathbb{Q}[x]$ having only simple roots to divide infinitely many standard quadrinomials or standard quintinomials over $\mathbb{Q}$. Moreover, we present a polynomial which yields negative answers to the questions of Győry and Schinzel and of Hajdu for $k=5$.

Theorem 1. A polynomial $P \in \mathbb{Q}[x]$ with only simple roots and with $P(0) \neq 0$ divides infinitely many standard quadrinomials with non-zero constant terms over $\mathbb{Q}$ if and only if either $P \in \mathrm{PR}_{4}$, or $P$ divides over $\mathbb{Q}$ two different standard quadrinomials with the same exponent quadruple. 
REMARK. We note that the tools in the proof of Theorem 1 can be used to derive the following effective version:

For every positive integer $d$ there exists an explicitly computable constant $C(d)$, depending only on $d$, such that a polynomial $P \in \mathbb{Q}[x]$ of degree $d$ with only simple roots and with $P(0) \neq 0$ divides more than $C(d)$ standard quadrinomials with non-zero constant terms over $\mathbb{Q}$ if and only if either $P \in \mathrm{PR}_{4}$, or $P$ divides over $\mathbb{Q}$ two different standard quadrinomials with the same exponent quadruple.

Theorem 2. A polynomial $P \in \mathbb{Q}[x]$ with only simple roots and with $P(0) \neq 0$ divides infinitely many standard quintinomials with non-zero constant terms over $\mathbb{Q}$ if and only if one of the following conditions holds:

(i) $P \in \mathrm{PR}_{5}$,

(ii) $P$ divides over $\mathbb{Q}$ two different standard quintinomials with the same exponent quintuple,

(iii) there exist integers $M_{1}, M_{2}, M_{3}, M_{4}$ such that $P$ divides over $\mathbb{Q}$ infinitely many standard quintinomials $Q_{M}$ of the form

$$
Q_{M}(x)=x^{M_{1}+2 M}+a_{M} x^{M_{2}+M}+b_{M} x^{M_{3}+M}+c_{M} x^{M_{4}+M}+d_{M},
$$

with $M \in \mathbb{N}$ and $a_{M}, b_{M}, c_{M}, d_{M} \in \mathbb{Q}$.

REMARK 1 . The condition that the constant terms are non-zero involves no serious restriction. Let $k$ be an integer with $k \geq 2, P \in \mathbb{Q}[x]$ with $P(0)=0$ be a polynomial and write $P(x)=x^{t} Q(x)$ with $t \geq 1$ and $Q(0) \neq 0$. Then $P$ divides infinitely many standard $k$-nomials over $\mathbb{Q}$ if and only if $Q$ divides a standard $(k-1)$-nomial over $\mathbb{Q}$. On the other hand, a polynomial $P \in \mathbb{Q}[x]$ with $P(0) \neq 0$ divides over $\mathbb{Q}$ infinitely many standard $k$-nomials with constant term zero if and only if $P$ divides a standard $(k-1)$-nomial over $\mathbb{Q}$. The proofs of these statements are trivial.

REMARK 2. We conjecture that in Theorem 1 the second condition implies the first, i.e. if some polynomial $P \in \mathbb{Q}[x]$ divides over $\mathbb{Q}$ two different standard quadrinomials with the same exponent quadruple, then $P \in \mathrm{PR}_{4}$. However, the similar condition (ii) in Theorem 2 does not imply that $P \in \mathrm{PR}_{5}$. This follows from the following proposition. The fact that $P$ does not divide any standard trinomial over $\mathbb{Q}$ yields a negative answer to the questions of Győry and Schinzel [2] and of Hajdu [3].

Proposition. The polynomial $P(x)=x^{5}-8 x^{2}-16 x+16$ divides infinitely many standard quintinomials over $\mathbb{Q}$ with the same exponent quintuple, but $P \notin \mathrm{PR}_{5}$ and $P$ does not divide any standard trinomial over $\mathbb{Q}$.

REMARK 3 . We do not know any example of a polynomial $P$ which satisfies condition (iii) of Theorem 2 without satisfying (i) or (ii). It is therefore not excluded that Theorem 2 is also valid if (iii) is omitted. It is even possible 
that for any $k \geq 5$ a polynomial $P \in \mathbb{Q}[x]$ with $P(0) \neq 0$ divides infinitely many standard $k$-nomials with non-zero constant terms over $\mathbb{Q}$ if and only if either $P \in \mathrm{PR}_{k}$ or $P$ divides over $\mathbb{Q}$ two standard $k$-nomials with the same exponent $k$-tuple.

2. Two basic lemmas. To prove Theorem 1 we need the following two lemmas, which play important roles also in the proof of Theorem 2. To formulate the first lemma, we need some preliminaries.

Let $\mathbb{K}$ be an algebraic number field and $\alpha_{i j} \in \mathbb{K}^{*}$ for $1 \leq i \leq m, 1 \leq$ $j \leq n$, where $m, n$ are positive integers. Moreover, let $a_{i} \in \mathbb{K}(1 \leq i \leq m)$. For $i=1, \ldots, m$ and $\underline{x} \in \mathbb{Z}^{n}$ with $\underline{x}=\left(x_{1}, \ldots, x_{n}\right)$ briefly write $\underline{\alpha_{i}} \underline{\underline{x}}=$ $\alpha_{i 1}^{x_{1}} \ldots \alpha_{i n}^{x_{n}}$. Consider the equation

$$
\sum_{i=1}^{m} a_{i} \underline{\alpha_{i}} \underline{\underline{x}}=0 \quad \text { in } \underline{x} \in \mathbb{Z}^{n} .
$$

Let $\mathcal{P}$ be a partition of the set $\Lambda=\{1, \ldots, m\}$, and consider the system of equations

$$
\sum_{i \in \lambda} a_{i} \underline{\alpha_{i}} \underline{\underline{x}}=0 \quad(\lambda \in \mathcal{P}) \quad \text { in } \underline{x} \in \mathbb{Z}^{n},
$$

which is a refinement of $(1)$. Let $\mathcal{S}(\mathcal{P})$ denote the set of those solutions of

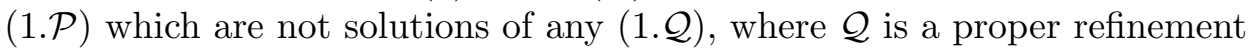
of $\mathcal{P}$. Set $i_{1} \stackrel{\mathcal{P}}{\sim} i_{2}$ if $i_{1}$ and $i_{2}$ are in the same class of $\mathcal{P}$, and put

$$
G(\mathcal{P})=\left\{\underline{z} \in \mathbb{Z}^{n}: \underline{\alpha_{i_{1}}} \underline{\underline{z}}=\underline{\alpha_{i_{2}}} \underline{z} \text { for any } i_{1}, i_{2} \text { with } i_{1} \mathcal{P} i_{2}\right\} .
$$

LEMMA 1. With the above notation, there exists an explicitly computable constant $C(m, n)$, depending only on $m$ and $n$, such that if $\mathcal{P}$ is any partition of $\Lambda$ with

$$
|\mathcal{S}(\mathcal{P})| \geq C(m, n),
$$

then there are different solutions $\underline{z}^{\prime}$ and $\underline{z}^{\prime \prime}$ of (1.P) such that $\underline{z}^{\prime}-\underline{z}^{\prime \prime} \in$ $G(\mathcal{P})$.

Proof. By induction, the statement is a simple consequence of Theorem 1.1 of $[1]$.

Lemma 2. Let $P \in \mathbb{Q}[x]$ be a polynomial with only simple roots and let $A$ be a set of standard k-nomials having the following property: for any two standard $k$-nomials in $A$ with exponent $k$-tuples $\left(m_{1}, \ldots, m_{k}\right)$ and $\left(m_{1}^{\prime}, \ldots, m_{k}^{\prime}\right)$, respectively, and for any $j_{1}, j_{2}, j_{3} \in\{1, \ldots, k\}$ with $j_{1}<j_{2}<$ $j_{3}$ the ordered pairs

$$
\left(m_{j_{1}}-m_{j_{3}}, m_{j_{2}}-m_{j_{3}}\right) \quad \text { and } \quad\left(m_{j_{1}}^{\prime}-m_{j_{3}}^{\prime}, m_{j_{2}}^{\prime}-m_{j_{3}}^{\prime}\right)
$$

are distinct. If $|A| \geq 2 \exp \left((6 k !)^{3 k !}\right)$ and $P$ divides all the $k$-nomials in $A$, then $P \in \mathrm{PR}_{k}$. 
Proof. The statement is a reformulation of the main result of Schlickewei and Viola [6].

\section{The quadrinomial case}

Proof of Theorem 1. If for some $P \in \mathbb{Q}[x]$ we have $P \in \mathrm{PR}_{4}$, then $P$ divides infinitely many standard quadrinomials over $\mathbb{Q}$ (see $[4$, pp. 339 and 348]). Moreover, if $P$ divides two standard quadrinomials $Q_{1}$ and $Q_{2}$ having the same exponent quadruple, then $P$ divides all the standard quadrinomials of the form

$$
\frac{a}{a+b} Q_{1}+\frac{b}{a+b} Q_{2}
$$

for every pair $(a, b)$ of positive rationals. Thus the "if" part of the theorem is proved.

Suppose that $P$ divides infinitely many standard quadrinomials with non-zero constant terms over $\mathbb{Q}$, and let $A$ be an infinite set of such standard quadrinomials. If there are infinitely many trinomials in $A$, then the statement of the theorem is a simple consequence of the Theorem of [5]. Hence we may assume that there are no trinomials in $A$. Moreover, in view of the second condition of the theorem, we may suppose that the exponent quadruples of the quadrinomials in $A$ are all distinct. Furthermore, omitting some elements of $A$ if necessary, we may also assume that the exponent quadruples of all the polynomials in $A$ are of the form

$$
\left(X, M_{2}, M_{3}, 0\right) \text { or } \quad\left(M_{1}+X, M_{2}+X, M_{3}+X, 0\right),
$$

where $M_{1}, M_{2}$ and $M_{3}$ are fixed integers. To verify this, first observe that for every $m$, there are only finitely many $Q \in A$ of degree $m$, since the exponent quadruples of the quadrinomials in $A$ are distinct. Thus we may assume, discarding some elements of $A$ if necessary, that there are no two polynomials in $A$ of the same degree. If $P \in \mathrm{PR}_{4}$, then the theorem holds. Otherwise let $B$ be a maximal subset of $A$ such that for any $Q, Q^{*}$ in $B$ with exponent quadruples $\left(m_{1}, m_{2}, m_{3}, 0\right)$ and $\left(m_{1}^{*}, m_{2}^{*}, m_{3}^{*}, 0\right)$, respectively, we have

$$
\left(m_{2}, m_{3}\right) \neq\left(m_{2}^{*}, m_{3}^{*}\right) \quad \text { and } \quad\left(m_{1}-m_{3}, m_{2}-m_{3}\right) \neq\left(m_{1}^{*}-m_{3}^{*}, m_{2}^{*}-m_{3}^{*}\right) .
$$

Using Lemma 2 we obtain $|B|<2 \exp \left(144^{72}\right)$. Then by the box principle we infer that for some $Q_{0} \in B$, with exponent quadruple $\left(M_{1}, M_{2}, M_{3}, 0\right)$ say, there are infinitely many polynomials $R$ in $A$ such that their exponent quadruples $\left(m_{1}, m_{2}, m_{3}, 0\right)$ satisfy

$$
\left(m_{2}, m_{3}\right)=\left(M_{2}, M_{3}\right) \quad \text { or } \quad\left(m_{1}-m_{3}, m_{2}-m_{3}\right)=\left(M_{1}-M_{3}, M_{2}-M_{3}\right),
$$

and our claim follows. 
Assume first that infinitely many $Q \in A$ have exponent quadruples of the form $\left(X, M_{2}, M_{3}, 0\right)$ with $M_{2}, M_{3}$ fixed. Omit every polynomial from $A$ which does not have this property.

We may suppose that $P$ has four pairwise non-equivalent roots $\alpha_{1}, \alpha_{2}$, $\alpha_{3}, \alpha_{4}$ under the relation introduced in the previous chapter, since otherwise we immediately obtain $P \in \mathrm{PR}_{4}$. As $P$ divides all the standard quadrinomials in $A$ over $\mathbb{Q}$, the equation

$$
\left|\begin{array}{cccc}
\alpha_{1}^{X} & \alpha_{2}^{X} & \alpha_{3}^{X} & \alpha_{4}^{X} \\
\alpha_{1}^{M_{2}} & \alpha_{2}^{M_{2}} & \alpha_{3}^{M_{2}} & \alpha_{4}^{M_{2}} \\
\alpha_{1}^{M_{3}} & \alpha_{2}^{M_{3}} & \alpha_{3}^{M_{3}} & \alpha_{4}^{M_{3}} \\
1 & 1 & 1 & 1
\end{array}\right|=0
$$

has infinitely many solutions in integers $X$ with $X>M_{2}$. By letting

$$
D_{i_{1} i_{2} i_{3}}=\left|\begin{array}{ccc}
\alpha_{i_{1}}^{M_{2}} & \alpha_{i_{2}}^{M_{2}} & \alpha_{i_{3}}^{M_{2}} \\
\alpha_{i_{1}}^{M_{3}} & \alpha_{i_{2}}^{M_{3}} & \alpha_{i_{3}}^{M_{3}} \\
1 & 1 & 1
\end{array}\right|
$$

for all $\left\{i_{1}, i_{2}, i_{3}\right\}$ with $1 \leq i_{1}<i_{2}<i_{3} \leq 4$, the previous equation can be rewritten as

$$
D_{234} \alpha_{1}^{X}-D_{134} \alpha_{2}^{X}+D_{124} \alpha_{3}^{X}-D_{123} \alpha_{4}^{X}=0 .
$$

Suppose that in (2) at least one of the subdeterminants $D_{i_{1} i_{2} i_{3}}$ does not vanish. Note that as the $\alpha_{i}$ 's are non-zero, at least two such determinants do not vanish in this case. Choose a partition $\mathcal{P}$ of the left hand side of (2) into subsums which vanish for infinitely many $X$ (belonging to the $Q$ 's in $A$ ), but have proper vanishing subsums only in finitely many instances. Omit all the $Q$ 's from $A$ for which these subsums either do not vanish, or have proper vanishing subsums. Observe that we still have $|A|=\infty$. Applying Lemma 1 to the partition $\mathcal{P}$, we find that for some non-zero integer $z_{1}$ and some indices $i_{1}, i_{2}$ with $1 \leq i_{1}<i_{2} \leq 4$,

$$
\alpha_{i_{1}}^{z_{1}}=\alpha_{i_{2}}^{z_{1}}
$$

which contradicts the non-equivalence of $\alpha_{i_{1}}$ and $\alpha_{i_{2}}$. This shows that all the determinants $D_{i_{1} i_{2} i_{3}}$ in (2) must vanish.

However, this yields that for some polynomials

$$
F_{j}(x)=x^{M_{2}}+a_{j} x^{M_{3}}+b_{j} \quad(j=1,2,3,4)
$$

with $a_{j}, b_{j} \in \mathbb{K},\left(a_{j}, b_{j}\right) \neq(0,0)$ we have

$$
F_{j}\left(\alpha_{i}\right)=0 \quad \text { for } i, j \in\{1,2,3,4\}, i \neq j,
$$

where $\mathbb{K}$ is the splitting field of $P$. Observe that if $F_{j} \neq F_{j^{\prime}}$ for some $j, j^{\prime}$, then there exist two $\alpha_{i}$ 's which are roots of the same binomial $F_{j}(x)-F_{j^{\prime}}(x)$, whence their ratio is a root of unity. This contradiction implies that $F_{1}=$ 
$F_{2}=F_{3}=F_{4}$, whence $\alpha_{1}, \alpha_{2}, \alpha_{3}, \alpha_{4}$ are roots of $F_{1}$. Note that $F_{1}$ is determined by $D_{234}$, which does not contain $\alpha_{1}$. If we take any other quadruple of pairwise non-equivalent $\alpha_{i}$ 's, the whole argument given above remains valid. We deduce by induction that every quadruple of non-equivalent $\alpha_{i}$ 's yield the same trinomial $F_{1}$. Hence, as $P$ has only simple roots, we may conclude that $P(x) \mid F_{1}(x)$ over $\mathbb{K}$. Suppose now that $\sigma\left(F_{1}\right) \neq F_{1}$ for some automorphism $\sigma$ of $\mathbb{K}$. As clearly $\sigma(P)=P$, this implies $P \mid \sigma\left(F_{1}\right)$ whence

$$
P \mid\left(F_{1}-\sigma\left(F_{1}\right)\right)
$$

over $\mathbb{K}$. However, the last polynomial is a binomial, and we deduce that the ratio of each pair of roots of $P$ is a root of unity, which is a contradiction. Hence we may assume that $\sigma\left(F_{1}\right)=F_{1}$ for all the automorphisms of $\mathbb{K}$. This immediately implies that $F_{1} \in \mathbb{Q}[x]$. If we observe that for any $Q \in A$ the polynomial $Q+F_{1}$ is a standard quadrinomial having the same exponent quadruple as $Q$, and that $P \mid Q+F_{1}$, the theorem follows in this case.

Assume now that infinitely many $Q \in A$ have exponent quadruples of the form $\left(M_{1}+X, M_{2}+X, M_{3}+X, 0\right)$ with $M_{1}, M_{2}, M_{3}$ fixed. This means that the polynomial $P$ divides standard quadrinomials of the shape

$$
x^{M_{1}+X}+a_{X} x^{M_{2}+X}+b_{X} x^{M_{3}+X}+c_{X} \quad \text { with } c_{X} \neq 0
$$

for infinitely many $X>0$. This is equivalent to saying that the polynomial $P^{*}(x)=x^{\operatorname{deg}(P)} P(1 / x)$ divides standard quadrinomials of the form $x^{M_{1}+X}+\left(b_{X} / c_{X}\right) x^{M_{1}-M_{3}}+\left(a_{X} / c_{X}\right) x^{M_{1}-M_{2}}+1 / c_{X}$ for infinitely many $X$ with $M_{1}+X>M_{1}-M_{3}$. However, for such polynomials $P^{*}$ the theorem has already been proved. Hence either $P^{*} \in \mathrm{PR}_{4}$ or $P^{*}$ divides two different standard quadrinomials having the same exponent quadruple $\left(m_{1}, m_{2}, m_{3}, 0\right)$. In the former case we infer that also $P \in \mathrm{PR}_{4}$, since the non-zero algebraic numbers $\alpha_{1}, \alpha_{2}, \alpha_{3}, \alpha_{4}$ are pairwise non-equivalent if and only if $1 / \alpha_{1}, 1 / \alpha_{2}, 1 / \alpha_{3}, 1 / \alpha_{4}$ are pairwise non-equivalent. In the latter case we obtain that $P$ divides two different standard quadrinomials with the same exponent quadruple $\left(m_{1}, m_{1}-m_{3}, m_{1}-m_{2}, 0\right)$ over $\mathbb{Q}$.

\section{The quintinomial case}

Lemma 3. Let $p$ and $q$ be integers with $p \neq 0, q \neq 0$ and $p \neq-q$, and $\Pi$ the set of the different permutations of $(p, q, 0,0,0)$. Let $\left(i_{1}, i_{2}, i_{3}, i_{4}, i_{5}\right)$ be any permutation of $(1,2,3,4,5)$, and put

$$
\begin{aligned}
& S_{1}=\left\{\left(i_{1}, i_{2}, i_{3}\right),\left(i_{1}, i_{2}, i_{4}\right),\left(i_{1}, i_{3}, i_{4}\right),\left(i_{2}, i_{3}, i_{4}\right)\right\}, \\
& S_{2}=\left\{\left(i_{1}, i_{2}, i_{3}\right),\left(i_{1}, i_{2}, i_{4}\right),\left(i_{1}, i_{2}, i_{5}\right),\left(i_{3}, i_{4}, i_{5}\right)\right\}, \\
& S_{3}=\left\{\left(i_{1}, i_{2}, i_{3}\right),\left(i_{1}, i_{2}, i_{4}\right),\left(i_{1}, i_{3}, i_{5}\right)\right\} .
\end{aligned}
$$

Let $S$ be any (possibly empty) subset of one of the sets $S_{i}(i=1,2,3)$, and let $\Pi^{*}$ be the set of those different permutations of $(p, q, 0,0,0)$ in which 
the index-triplets of the places of the 0's belong to $S$. Put $T=\Pi \backslash \Pi^{*}$, and let $T_{1}, \ldots, T_{t}$ be any partition of $T$ with $\left|T_{i}\right| \geq 2(1 \leq i \leq t)$. Let $H$ be the subspace of $\mathbb{Q}^{5}$ generated by the set of quintuples $\bigcup_{i=1}^{t} \bigcup_{u, v \in T_{i}}\{u-v\}$ over $\mathbb{Q}$. Then $H$ contains a quintuple having exactly two non-zero entries.

Proof. Note that if the statement is true for some choice of $\left(i_{1}, i_{2}, i_{3}\right.$, $\left.i_{4}, i_{5}\right)$, then it is clearly true for the other permutations of $(1,2,3,4,5)$. Throughout the proof, we call a quintuple nice if it has exactly two non-zero entries. We split the proof of the lemma into several parts.

Assume that $p=q$. Clearly, without loss of generality we may suppose that $p=q=1$. If two elements from $T$ are in some $T_{i}$ with one 1 at the same place, then we are done. Since there are only five entries, we are also done if $\left|T_{i}\right| \geq 3$ for some $i$. So $\left|T_{i}\right|=2$ for every $i$ and $|T|$ cannot be odd. By symmetry we may assume that $T_{1}=\{(1,1,0,0,0),(0,0,1,1,0)\}$ with difference vector $v_{1}=(1,1,-1,-1,0)$. If there is another class with elements having 0 's at the last entry, then we may assume without loss of generality that $T_{2}=\{(1,0,1,0,0),(0,1,0,1,0)\}$ with difference vector $v_{0}=(1,-1,1,-1,0)$ and then $v_{1}+v_{0}=(2,0,0,-2,0)$ is a nice quintuple in $H$. Otherwise we can permute the entries in such a way that $T_{2}=\{(1,0,0,0,1),(0,1,1,0,0)\}$ with difference vector $v_{2}=(1,-1,-1,0,1)$. Since $|S| \leq 4$, we know that there is a third class $T_{3}$. There are only five possible additional classes, yielding the difference vectors $v_{3}=(1,-1,1,0,-1), v_{4}=(1,0,1,-1,-1)$, $v_{5}=(1,-1,0,1,-1), v_{6}=(1,0,-1,1,-1), v_{7}=(0,1,-1,1,-1)$, respectively. In the first case $v_{2}+v_{3}$ is nice, and in the fourth case $2 v_{1}-v_{2}-v_{6}$ is nice. In the remaining three cases the places of the 0's do not belong to $S_{1}$ or $S_{2}$. We conclude that $|S| \leq 3,|T| \geq 7$ and there should be a fourth pair. However, the quintuples $v_{1}-3 v_{2}-2 v_{4}+4 v_{5}, 3 v_{1}-v_{2}-2 v_{4}-4 v_{7}$ and $v_{1}+v_{2}-2 v_{5}-2 v_{7}$ are all nice. This proves the lemma in the case of $p=q$.

Suppose now that $p \neq q$ and that the subspace $H$ does not contain any nice quintuple. Clearly, we may assume that $p>|q|$ and $\operatorname{gcd}(p, q)=1$. Observe that for any $\left(x_{1}, x_{2}, x_{3}, x_{4}, x_{5}\right) \in H$ we have

$$
x_{1}+x_{2}+x_{3}+x_{4}+x_{5}=0 .
$$

If the dimension of $H$ is 4 , then starting from a basis of $H$ over $\mathbb{Q}$, by Gaussian elimination we can obtain a nice vector in $H$. Thus we may suppose that another linear relation is also valid for the elements of $H$, i.e.

$$
\lambda_{1} x_{1}+\lambda_{2} x_{2}+\lambda_{3} x_{3}+\lambda_{4} x_{4}+\lambda_{5} x_{5}=0
$$

holds for every $\left(x_{1}, x_{2}, x_{3}, x_{4}, x_{5}\right) \in H$, where $\lambda_{i} \in \mathbb{Q}(i=1, \ldots, 5)$, and these coefficients are not all equal. Plainly, we may assume that $\lambda_{i} \in \mathbb{Z}$ $(i=1, \ldots, 5)$ and $\operatorname{gcd}\left(\lambda_{1}, \lambda_{2}, \lambda_{3}, \lambda_{4}, \lambda_{5}\right)=1$, moreover, that $\max \left|\lambda_{i}-\lambda_{j}\right|$ is minimal among all such coefficient quintuples. Put

$$
I=\left\{i \in\{1,2,3,4,5\}: y_{i} \neq 0 \text { for some }\left(y_{1}, y_{2}, y_{3}, y_{4}, y_{5}\right) \in T\right\} .
$$


Let now $Y=\left(y_{1}, y_{2}, y_{3}, y_{4}, y_{5}\right)$ and $Z=\left(z_{1}, z_{2}, z_{3}, z_{4}, z_{5}\right)$ be two quintuples from the same class of $T$, and suppose that $y_{i}=z_{j}=q$. If $i=j$, then $Y-Z$ is a nice quintuple in $H$. If $i \neq j$, then from $Y-Z \in H$ by $\operatorname{gcd}(p, q)=1$ we have $\lambda_{i} \equiv \lambda_{j} \bmod p$. Now if all the $\lambda_{i}$ with $i \in I$ belong to the same residue class $r$ mod $p$, then by the relations (3) and (4) we deduce that

$$
\sum_{i \in I}\left(\lambda_{i}-r\right) x_{i}=0
$$

is valid for every $\left(x_{1}, x_{2}, x_{3}, x_{4}, x_{5}\right) \in H$. However, after dividing by the gcd of the coefficients (which is $\geq p$ ), we get a coefficient quintuple $\left(\lambda_{1}^{\prime}, \lambda_{2}^{\prime}, \lambda_{3}^{\prime}, \lambda_{4}^{\prime}, \lambda_{5}^{\prime}\right)$, for which (4) is valid and

$$
\max \left|\lambda_{i}^{\prime}-\lambda_{j}^{\prime}\right|<\max \left|\lambda_{i}-\lambda_{j}\right|,
$$

which is a contradiction. Thus the set $I$ splits over two classes $I_{1}$ and $I_{2}$ with $\left|I_{2}\right| \geq\left|I_{1}\right| \geq 2$, such that if $i, j \in I_{k}$, then $\lambda_{i} \equiv \lambda_{j} \bmod p(k=1,2)$. Moreover, using (3) if necessary, we may assume that $\lambda_{i} \equiv 0 \bmod p$ for every $i \in I_{2}$.

Suppose that $|I| \leq 4$. Then $S=S_{1}$ for some permutation $\left(i_{1}, i_{2}, i_{3}, i_{4}, i_{5}\right)$ of $(1,2,3,4,5)$. Without loss of generality we may assume that $\left(i_{1}, i_{2}, i_{3}, i_{4}, i_{5}\right)$ $=(1,2,3,4,5)$ and

$$
\lambda_{1} \equiv \lambda_{2} \not \equiv \lambda_{3} \equiv \lambda_{4} \equiv 0 \bmod p .
$$

Observe that in this case $|T|=12$. It is also clear that every class $T_{j}$ has two elements. Without loss of generality we may assume

$$
T_{1}=\{(q, p, 0,0,0),(0, q, p, 0,0)\} .
$$

If

$$
T_{2}=\{(q, 0, p, 0,0),(p, q, 0,0,0)\},
$$

then we have a class

$$
T_{3}=\{(q, 0,0, p, 0),(0, q, 0, p, 0)\},
$$

and we get the nice vector

$$
(q,-q, 0,0,0)=(q, 0,0, p, 0)-(0, q, 0, p, 0)
$$

in $H$. Thus we may suppose that

$$
T_{2}=\{(q, 0, p, 0,0),(0, q, 0, p, 0)\} \quad \text { and } \quad T_{3}=\{(q, 0,0, p, 0),(p, q, 0,0,0)\} .
$$

Applying (4) for the difference of the vectors in $T_{2}$, we get

$$
\lambda_{1} q-\lambda_{2} q+\lambda_{3} p-\lambda_{4} p=0 .
$$

By (5) and $\operatorname{gcd}(p, q)=1$ this implies $\lambda_{1} \equiv \lambda_{2} \bmod p^{2}$. On the other hand, for the difference of the vectors in $T_{1}$, (4) yields

$$
\lambda_{1} q+\lambda_{2}(p-q)-\lambda_{3} p=0 .
$$


With the use of $\lambda_{1} \equiv \lambda_{2} \bmod p^{2}$ and (5), this gives $\lambda_{2} \equiv 0 \bmod p$, which is a contradiction.

So we may assume that $|I|=5$ and

$$
\lambda_{1} \equiv \lambda_{2} \not \equiv \lambda_{3} \equiv \lambda_{4} \equiv \lambda_{5} \equiv 0 \bmod p \text {. }
$$

Clearly,

$$
|T|=\#_{1}+\#_{2}+\#_{3}+\#_{4}+\#_{5},
$$

where $\#_{i}$ denotes the number of quintuples in $T$ with $q$ at the $i$ th position $(i=1, \ldots, 5)$. As we consider quintuples, plainly $\#_{i} \leq 4$. Moreover, observe that $4-\#_{i}$ equals the number of triplets in $S$ in which $i$ does not occur.

By $(6), \#_{1}=\#_{2}$. We claim that $\#_{1}+\#_{2} \leq 4$. To prove this, suppose that there are two classes $T_{1}$ and $T_{2}$, in which the places of the $q$ belong to $\{1,2\}$, in $T_{1}$ a $p$ occurs at a position from $\{1,2\}$, and the other $p$ 's in $T_{1}$ and $T_{2}$ are at positions from $\{3,4,5\}$. Applying (4) for the difference of the quintuples in $T_{2}$ and using $\operatorname{gcd}(p, q)=1$ and $(6)$, we obtain $\lambda_{1} \equiv \lambda_{2} \bmod p^{2}$. If we now apply (4) for the difference of the vectors in $T_{1}$, by $\operatorname{gcd}(p, q)=1$ and $(6)$ the previous congruence yields $\lambda_{1} \equiv \lambda_{2} \equiv 0 \bmod p$, which is a contradiction. Thus, if $\#_{1}+\#_{2} \geq 6$, then without loss of generality we may assume that three classes are given by

$$
\begin{gathered}
T_{1}=\{(q, 0, p, 0,0),(0, q, 0, p, 0)\}, \quad T_{2}=\{(q, 0,0, p, 0),(0, q, 0,0, p)\}, \\
T_{3}=\{(q, 0,0,0, p),(0, q, p, 0,0)\} .
\end{gathered}
$$

However, now the sum of the three difference vectors provided by $T_{1}, T_{2}, T_{3}$ gives a nice quintuple in $H$. This proves $\#_{1}+\#_{2} \leq 4$. Hence $|T| \leq 16$ and $|S| \geq 2$.

Suppose that $|T|=16$. Then $\#_{3}+\#_{4}+\#_{5}=12$. This implies $\#_{i}=4$ for $i=3,4,5$, whence $i \in s$ for every triplet $s \in S(i=3,4,5)$. However, as $|S|>1$, this is impossible. Thus $|T| \leq 14$ and $|S| \geq 3$.

Suppose $|T|=14$. As we just proved that $\#_{3}+\#_{4}+\#_{5}=12$ is impossible, we must have $\#_{3}+\#_{4}+\#_{5}=10$. This implies that in the triplets of $S$, $3,4,5$ altogether are missing exactly twice. Thus without loss of generality we may assume that $S$ is given by one of the sets

$$
\begin{gathered}
\{(3,4,5),(1,3,5),(2,3,5)\}, \quad\{(3,4,5),(1,3,5),(1,4,5)\}, \\
\{(3,4,5),(1,3,5),(2,4,5)\} .
\end{gathered}
$$

In the first case the quintuples with $q$ at places 1 or 2 are given by

$$
(q, 0, p, 0,0), \quad(q, 0,0,0, p), \quad(0, q, p, 0,0), \quad(0, q, 0,0, p) .
$$

It is easy to check that no matter how we distribute these vectors into two classes $T_{1}$ and $T_{2}$, we get a nice quintuple in $H$.

In the second case the quintuples with $q$ at the places 1 or 2 are given by

$$
(q, 0, p, 0,0), \quad(q, 0,0, p, 0), \quad(q, 0,0,0, p), \quad(0, q, 0,0, p),
$$

and one of the difference vectors is nice. 
The third case is somewhat more complicated. In fact now we have $S=$ $S_{3}$ with $\left(i_{1}, i_{2}, i_{3}, i_{4}, i_{5}\right)=(5,3,4,2,1)$. Observe that two classes must be given by

$$
T_{1}=\{(q, 0,0, p, 0),(0, q, 0,0, p)\}, \quad T_{2}=\{(q, 0,0,0, p),(0, q, p, 0,0)\} .
$$

Thus the quintuple

$$
\frac{1}{p}[(q, 0,0, p, 0)-(0, q, 0,0, p)+(0, q, p, 0,0)-(q, 0,0,0, p)]=(0,0,1,1,-2)
$$

is in $H$. Further, consider the classes $T_{3}$ and $T_{4}$ that contain the quintuples $(0,0, p, 0, q)$ and $(0,0,0, p, q)$, respectively. If one of the vectors

$$
(p, 0,0,0, q), \quad(0, p, 0,0, q)
$$

belongs to $T_{3}$ or $T_{4}$, then we get a nice difference vector. If any of the quintuples

$$
(0,0, q, p, 0), \quad(0,0, q, 0, p), \quad(0,0, p, q, 0), \quad(0,0,0, q, p)
$$

of $T$ is either in $T_{3}$ or $T_{4}$, then with the help of $(0,0,1,1,-2) \in H$ and $p>|q|$ we again obtain a nice vector in $H$. So we have either

$$
T_{3}=\{(0,0, p, 0, q),(0, p, q, 0,0)\} \quad \text { and } \quad T_{4}=\{(0,0,0, p, q),(p, 0,0, q, 0)\},
$$

or

$$
T_{3}=\{(0,0, p, 0, q),(p, 0,0, q, 0)\} \quad \text { and } \quad T_{4}=\{(0,0,0, p, q),(0, p, q, 0,0)\} .
$$

In the first case $T_{1}$ and $T_{2}$ yield a vector $(2 q,-2 q,-p, p, 0) \in H$, and $T_{3}$ and $T_{4}$ a vector $(p,-p, p-q, q-p, 0) \in H$. A linear combination of these quintuples is given by the nice vector

$$
\left(0,0, p^{2}+2 p q-2 q^{2},-p^{2}-2 p q+2 q^{2}, 0\right) \in H .
$$

In the second case we obtain a nice quintuple in $H$ in a similar way. Thus $|T| \leq 12$ and $|S| \geq 4$.

We conclude that $|T|=12$ and $|S|=4$. Since $S=S_{1}$ is already excluded, we have $S=S_{2}$ for some permutation $\left(i_{1}, i_{2}, i_{3}, i_{4}, i_{5}\right)$ of $(1,2,3,4,5)$. By $\#_{1}+\#_{2} \leq 4$, we find $1,2 \in\left\{i_{3}, i_{4}, i_{5}\right\}$. Because of symmetry we may assume without loss of generality that $\left(i_{1}, i_{2}, i_{3}, i_{4}, i_{5}\right)=(4,5,1,2,3)$. Hence the quintuples with $q$ at the places 1 or 2 are given by

$$
(q, 0,0, p, 0), \quad(q, 0,0,0, p), \quad(0, q, 0, p, 0), \quad(0, q, 0,0, p) .
$$

It is easy to check that no matter how we distribute these vectors into two classes, we get a nice quintuple in $H$.

In the proof of the following statement we need some concepts of graph theory. In what follows, $V$ will denote a graph, and sometimes also the set of vertices of this graph. If $V^{\prime}$ is a set of vertices of $V$, the restriction of $V$ 
to $V^{\prime}$ means the graph with vertices in $V^{\prime}$ and with those edges of $V$ for which there is a corresponding edge in $V^{\prime}$.

Lemma 4. Let $P \in \mathbb{Q}[x]$ with $P(0) \neq 0$ be a polynomial having only simple roots and assume that among these roots there are at least five which are pairwise non-equivalent. Suppose that $P$ divides over $\mathbb{Q}$ infinitely many standard quintinomials with distinct exponent quintuples and non-zero constant terms, and let $A$ be an infinite set of such standard quintinomials. Furthermore, suppose that for the exponent quintuples $\left(m_{1}, m_{2}, m_{3}, m_{4}, 0\right)$ and $\left(m_{1}^{\prime}, m_{2}^{\prime}, m_{3}^{\prime}, m_{4}^{\prime}, 0\right)$ of any pair $Q_{1}, Q_{2} \in A$ we have

and either

$$
m_{1} \neq m_{1}^{\prime}, \quad m_{2} \neq m_{2}^{\prime}
$$

$$
\left(m_{3}, m_{4}\right)=\left(m_{3}^{\prime}, m_{4}^{\prime}\right)
$$

or

$$
m_{2}-m_{2}^{\prime}=m_{3}-m_{3}^{\prime}=m_{4}-m_{4}^{\prime}, \quad \frac{m_{1}-m_{1}^{\prime}}{m_{2}-m_{2}^{\prime}} \notin\{1,2\} .
$$

Put $\left(r_{1}, r_{2}\right)=\left(m_{3}, m_{4}\right)$ and $\left(r_{1}, r_{2}\right)=\left(m_{2}-m_{4}, m_{3}-m_{4}\right)$ in case (i) and (ii), respectively. Define the graph $V$ in the following way. Let the vertices of $V$ be the non-ordered triplets $\left\{\alpha_{i_{1}}, \alpha_{i_{2}}, \alpha_{i_{3}}\right\}$ of pairwise nonequivalent roots of $P$ for which the determinant

$$
\left|\begin{array}{ccc}
\alpha_{i_{1}}^{r_{1}} & \alpha_{i_{2}}^{r_{1}} & \alpha_{i_{3}}^{r_{1}} \\
\alpha_{i_{1}}^{r_{2}} & \alpha_{i_{2}}^{r_{2}} & \alpha_{i_{3}}^{r_{2}} \\
1 & 1 & 1
\end{array}\right|
$$

vanishes. Connect two vertices $v_{1}$ and $v_{2}$ by an edge if and only if $\left|v_{1} \cap v_{2}\right|$ $=2$. Then the graph $V$ is connected and for every root $\alpha$ of $P$ there exists $a v \in V$ such that $\alpha \in v$.

Proof. Observe that to prove the statement, it is sufficient to verify that under the assumptions of the lemma the following three conditions hold:

(a) for any set of pairwise non-equivalent roots $L=\left\{\alpha_{1}, \alpha_{2}, \alpha_{3}, \alpha_{4}, \alpha_{5}\right\}$ of $P$ the subgraph $V_{L}$ of $V$ whose vertices are the triplets of $V$ with entries from $L$ has the property that for every $i$ with $1 \leq i \leq 5$ there exists a $v \in V_{L}$ such that $\alpha_{i} \in v$,

(b) the above-defined subgraphs $V_{L}$ are all connected,

(c) if both sets $L=\left\{\alpha_{1}, \alpha_{2}, \alpha_{3}, \alpha_{4}, \alpha_{5}\right\}$ and $M=\left\{\alpha_{1}^{*}, \alpha_{2}, \alpha_{3}, \alpha_{4}, \alpha_{5}\right\}$ consist of pairwise non-equivalent roots of $P$, then the restriction of $V$ to $V_{L} \cup V_{M}$ is connected.

Indeed, (a) implies that for every root $\alpha$ of $P$ there is some $v \in V$ such that $\alpha \in v$, while (b) and (c) together yield that $V$ is connected.

Moreover, (c) can be replaced by the condition 
( $\left.\mathrm{c}^{\prime}\right)$ for any set of pairwise non-equivalent roots $L=\left\{\alpha_{1}, \alpha_{2}, \alpha_{3}, \alpha_{4}, \alpha_{5}\right\}$ of $P$ the corresponding subgraph $V_{L}$ satisfies

$$
V_{L} \neq\left\{\left\{\alpha_{i_{1}}, \alpha_{i_{2}}, \alpha_{i_{3}}\right\},\left\{\alpha_{i_{1}}, \alpha_{i_{2}}, \alpha_{i_{4}}\right\},\left\{\alpha_{i_{1}}, \alpha_{i_{3}}, \alpha_{i_{5}}\right\}\right\}
$$

for any permutation $\left(i_{1}, i_{2}, i_{3}, i_{4}, i_{5}\right)$ of $(1,2,3,4,5)$.

To prove this, assume that (a), (b) and ( $\left.\mathrm{c}^{\prime}\right)$ are true for both $L=$ $\left\{\alpha_{1}, \alpha_{2}, \alpha_{3}, \alpha_{4}, \alpha_{5}\right\}$ and $M=\left\{\alpha_{1}^{*}, \alpha_{2}, \alpha_{3}, \alpha_{4}, \alpha_{5}\right\}$ consisting of pairwise nonequivalent roots of $P$, but (c) does not hold. Suppose that there is a $v \in V_{M}$ of the form $v=\left\{\alpha_{j_{1}}, \alpha_{j_{2}}, \alpha_{j_{3}}\right\}$ with $2 \leq j_{1}<j_{2}<j_{3} \leq 5$. Then $v \in V_{L}$, and (c) holds. Thus to avoid (c),

$$
V_{M} \subseteq \bigcup_{\substack{\left(j_{2}, j_{3}\right) \\ 2 \leq j_{2}<j_{3} \leq 5}}\left\{\left\{\alpha_{1}^{*}, \alpha_{j_{2}}, \alpha_{j_{3}}\right\}\right\}
$$

and by symmetry,

$$
V_{L} \subseteq \bigcup_{\substack{\left(j_{2}, j_{3}\right) \\ 2 \leq j_{2}<j_{3} \leq 5}}\left\{\left\{\alpha_{1}, \alpha_{j_{2}}, \alpha_{j_{3}}\right\}\right\}
$$

must be valid. To preserve (a) and (b) but avoid (c), here

$$
V_{M}=\left\{\left\{\alpha_{1}^{*}, \alpha_{j_{2}}, \alpha_{j_{3}}\right\},\left\{\alpha_{1}^{*}, \alpha_{j_{2}}, \alpha_{j_{5}}\right\},\left\{\alpha_{1}^{*}, \alpha_{j_{4}}, \alpha_{j_{5}}\right\}\right\}
$$

together with

$$
V_{L}=\left\{\left\{\alpha_{1}, \alpha_{j_{2}}, \alpha_{j_{4}}\right\},\left\{\alpha_{1}, \alpha_{j_{3}}, \alpha_{j_{5}}\right\},\left\{\alpha_{1}, \alpha_{j_{3}}, \alpha_{j_{4}}\right\}\right\}
$$

should hold, where $\left\{j_{2}, j_{3}, j_{4}, j_{5}\right\}=\{2,3,4,5\}$. However, in this case we find that $\left(\mathrm{c}^{\prime}\right)$ is not valid for $V_{L}$ by choosing

$$
\left(i_{1}, i_{2}, i_{3}, i_{4}, i_{5}\right)=\left(1, j_{3}, j_{4}, j_{5}, j_{2}\right) .
$$

This shows that (a), (b) and ( $\left.c^{\prime}\right)$ together imply (c).

We prove the lemma under the assumption (i) first. Put $\left(M_{3}, M_{4}\right)=$ $\left(m_{3}, m_{4}\right)$. Assume that one of the conditions (a), (b) or $\left(\mathrm{c}^{\prime}\right)$ does not hold, and fix a set $L=\left\{\alpha_{1}, \alpha_{2}, \alpha_{3}, \alpha_{4}, \alpha_{5}\right\}$ of pairwise non-equivalent roots of $P$ such that this happens also for this set. For all $\left\{i_{1}, i_{2}, i_{3}\right\}$ with $1 \leq i_{1}<i_{2}<$ $i_{3} \leq 5$ put

$$
D_{i_{1} i_{2} i_{3}}=\left|\begin{array}{ccc}
\alpha_{i_{1}}^{M_{3}} & \alpha_{i_{2}}^{M_{3}} & \alpha_{i_{3}}^{M_{3}} \\
\alpha_{i_{1}}^{M_{4}} & \alpha_{i_{2}}^{M_{4}} & \alpha_{i_{3}}^{M_{4}} \\
1 & 1 & 1
\end{array}\right| .
$$

Suppose first that (a) is not valid, that is, for some $i$ with $1 \leq i \leq 5$ and for every element $v$ of the corresponding subgraph $V_{L}$ of $V$, we have $\alpha_{i} \notin v$. Without loss of generality we may assume that here $i=5$. By our assumptions we have

$$
V_{L} \subseteq\left\{\left\{\alpha_{1}, \alpha_{2}, \alpha_{3}\right\},\left\{\alpha_{1}, \alpha_{2}, \alpha_{4}\right\},\left\{\alpha_{1}, \alpha_{3}, \alpha_{4}\right\},\left\{\alpha_{2}, \alpha_{3}, \alpha_{4}\right\}\right\} .
$$


Let $I$ be the set of the index-triplets of the vanishing determinants $D_{i_{1} i_{2} i_{3}}$. We clearly have

$$
I \subseteq\{\{1,2,3\},\{1,2,4\},\{1,3,4\},\{2,3,4\}\} .
$$

At this point we split our proof into two parts.

1) Suppose that we also have

$$
m_{1}-m_{2} \neq m_{1}^{\prime}-m_{2}^{\prime}
$$

for every pair $Q_{1}, Q_{2}$ in $A$, up to finitely many exceptions. Note that we may also assume that

$$
m_{1}+m_{2} \neq m_{1}^{\prime}+m_{2}^{\prime}
$$

for all $Q_{1}, Q_{2} \in A$. Indeed, for every $N \in \mathbb{N}$ there can be at most finitely many $Q_{1} \in A$ with $m_{1}+m_{2}=N$, otherwise there would be infinitely many polynomials in $A$ with the same exponent quintuple.

The fact that $P$ divides all the standard quintinomials in $A$ means that the equation

$$
\left|\begin{array}{ccccc}
\alpha_{1}^{M_{1}} & \alpha_{2}^{M_{1}} & \alpha_{3}^{M_{1}} & \alpha_{4}^{M_{1}} & \alpha_{5}^{M_{1}} \\
\alpha_{1}^{M_{2}} & \alpha_{2}^{M_{2}} & \alpha_{3}^{M_{2}} & \alpha_{4}^{M_{2}} & \alpha_{5}^{M_{2}} \\
\alpha_{1}^{M_{3}} & \alpha_{2}^{M_{3}} & \alpha_{3}^{M_{3}} & \alpha_{4}^{M_{3}} & \alpha_{5}^{M_{3}} \\
\alpha_{1}^{M_{4}} & \alpha_{2}^{M_{4}} & \alpha_{3}^{M_{4}} & \alpha_{4}^{M_{4}} & \alpha_{5}^{M_{4}} \\
1 & 1 & 1 & 1 & 1
\end{array}\right|=0
$$

has infinitely many solutions in integers $M_{1}, M_{2}$ with $M_{1}>M_{2}>M_{3}$, for any fixed set $\left\{\alpha_{1}, \alpha_{2}, \alpha_{3}, \alpha_{4}, \alpha_{5}\right\}$ of pairwise non-equivalent roots of $P$. The equation can be rewritten as

$$
\sum_{\substack{\left\{i_{1}, i_{2}, i_{3}, i_{4}, i_{5}\right\}=\{1,2,3,4,5\} \\ i_{3}<i_{4}<i_{5}}}(-1)^{i_{1}+i_{2}+1} \operatorname{sgn}\left(i_{2}-i_{1}\right) D_{i_{3} i_{4} i_{5}} \alpha_{i_{1}}^{M_{1}} \alpha_{i_{2}}^{M_{2}}=0 .
$$

Let

$$
\sum_{\substack{\left\{i_{1}, i_{2}, i_{3}, i_{4}, i_{3}\right\}=\{1,2,3,4,5\} \\ i_{3}<i_{4}<i_{5}}}^{*}(-1)^{i_{1}+i_{2}+1} \operatorname{sgn}\left(i_{2}-i_{1}\right) D_{i_{3} i_{4} i_{5}} \alpha_{i_{1}}^{M_{1}} \alpha_{i_{2}}^{M_{2}}=0
$$

be the equation obtained by removing the terms with $D_{i_{3} i_{4} i_{5}}=0$. Choose a system $\mathcal{P}$ of subsums of the left hand side of (7) such that each subsum in $\mathcal{P}$ vanishes for infinitely many exponent quintuples corresponding to the polynomials in $A$ simultaneously, but all the proper subsums of these subsums do not vanish. Remove all other polynomials from $A$. Clearly, we still have $|A|=\infty$. Applying Lemma 1 to the partition $\mathcal{P}$, we obtain $G(\mathcal{P}) \neq\{\underline{0}\}$. Hence for some $\underline{z}=\left(z_{1}, z_{2}\right) \in \mathbb{Z}^{2}$ with $\left(z_{1}, z_{2}\right) \neq(0,0)$ and for all $\left(i_{1}, i_{2}\right),\left(j_{1}, j_{2}\right)$ we see that if $\alpha_{i_{1}}^{M_{1}} \alpha_{i_{2}}^{M_{2}}$ and $\alpha_{j_{1}}^{M_{1}} \alpha_{j_{2}}^{M_{2}}$ occur in the same 
class of $\mathcal{P}$, then

$$
\alpha_{i_{1}}^{z_{1}} \alpha_{i_{2}}^{z_{2}}=\alpha_{j_{1}}^{z_{1}} \alpha_{j_{2}}^{z_{2}}
$$

Thus we obtain a lot of multiplicative relations among the $\alpha_{i}$ 's, and to get a contradiction it suffices to prove that these relations imply that the ratio of two such roots is a root of unity. Put $p=z_{1}$ and $q=z_{2}$, and observe that by our assumptions

$m_{1} \neq m_{1}^{\prime}, \quad m_{2} \neq m_{2}^{\prime}, \quad m_{1}-m_{2} \neq m_{1}^{\prime}-m_{2}^{\prime} \quad$ and $\quad m_{1}+m_{2} \neq m_{1}^{\prime}+m_{2}^{\prime}$, as $\left(z_{1}, z_{2}\right)$ comes from the difference of two distinct solutions $\left(M_{1}, M_{2}\right)$ and $\left(M_{1}^{\prime}, M_{2}^{\prime}\right)$ of $(7)$, we may suppose that

$$
p \neq 0, \quad q \neq 0, \quad p \neq q \text { and } \quad p \neq-q .
$$

We apply Lemma 3 with $S=S_{1}$ to the partition $\mathcal{P}=\left\{T_{1}, \ldots, T_{t}\right\}$ corresponding to our vanishing subsums in (7). Note that the inequality $p \neq q$ implies that $|\Pi|=20$. We deduce that from our multiplicative relations it is possible to derive a relation in which exactly two roots are involved, and we may assume that their exponents are integers. Moreover as the sum of the entries of every vector in $H$ (defined in Lemma 3 ) is zero, in this relation the sum of the two non-zero exponents equals 0 . Hence the ratio of the two roots is a root of unity. This contradiction shows that (a) must hold in case 1).

2) Suppose that for infinitely many polynomials in $A$ we have

$$
m_{1}-m_{2}=m_{1}^{\prime}-m_{2}^{\prime}
$$

Remove all the other standard quintinomials from $A$. For the remaining polynomials in $A$ put $\left(M_{3}, M_{4}\right)=\left(m_{3}, m_{4}\right)$ and $M=m_{1}-m_{2}$, and observe that the equation

$$
\left|\begin{array}{ccccc}
\alpha_{1}^{M_{2}+M} & \alpha_{2}^{M_{2}+M} & \alpha_{3}^{M_{2}+M} & \alpha_{4}^{M_{2}+M} & \alpha_{5}^{M_{2}+M} \\
\alpha_{1}^{M_{2}} & \alpha_{2}^{M_{2}} & \alpha_{3}^{M_{2}} & \alpha_{4}^{M_{2}} & \alpha_{5}^{M_{2}} \\
\alpha_{1}^{M_{3}} & \alpha_{2}^{M_{3}} & \alpha_{3}^{M_{3}} & \alpha_{4}^{M_{3}} & \alpha_{5}^{M_{3}} \\
\alpha_{1}^{M_{4}} & \alpha_{2}^{M_{4}} & \alpha_{3}^{M_{4}} & \alpha_{4}^{M_{4}} & \alpha_{5}^{M_{4}} \\
1 & 1 & 1 & 1 & 1
\end{array}\right|=0
$$

has infinitely many solutions in integers $M_{2}$ with $M_{2}>M_{3}$. The equation can be rewritten as

$$
\sum_{\substack{\left\{i_{1}, i_{2}, i_{3}, i_{4}, i_{5}\right\}=\{1,2,3,4,5\} \\ i_{1}<i_{2}, i_{3}<i_{4}<i_{5}}}(-1)^{i_{1}+i_{2}+1}\left(\alpha_{i_{1}}^{M}-\alpha_{i_{2}}^{M}\right) D_{i_{3} i_{4} i_{5}}\left(\alpha_{i_{1}} \alpha_{i_{2}}\right)^{M_{2}}=0 .
$$

Observe that for any $1 \leq i_{1}<i_{2} \leq 5$, the non-equivalence of $\alpha_{i_{1}}$ and $\alpha_{i_{2}}$ implies that

$$
\alpha_{i_{1}}^{M}-\alpha_{i_{2}}^{M} \neq 0
$$


Let

$$
\sum_{\substack{\left\{i_{1}, i_{2}, i_{3}, i_{4}, i_{5}\right\}=\{1,2,3,4,5\} \\ i_{1}<i_{2}, i_{3}<i_{4}<i_{5}}}^{*}(-1)^{i_{1}+i_{2}+1}\left(\alpha_{i_{1}}^{M}-\alpha_{i_{2}}^{M}\right) D_{i_{3} i_{4} i_{5}}\left(\alpha_{i_{1}} \alpha_{i_{2}}\right)^{M_{2}}=0
$$

be the equality obtained after removing the terms with $D_{i_{3} i_{4} i_{5}}=0$. Choose a system $\mathcal{P}$ of subsums of the left hand side of (8) such that each subsum in $\mathcal{P}$ vanishes for infinitely many exponent quintuples corresponding to the polynomials in $A$ simultaneously, but all the proper subsums of these subsums do not vanish. Remove all the other polynomials from $A$. Clearly, we still have $|A|=\infty$. Applying Lemma 1 to the partition $\mathcal{P}$, we obtain $G(\mathcal{P}) \neq\{\underline{0}\}$. Hence for some $z_{1} \in \mathbb{Z}$ with $z_{1} \neq 0$ and for all $\left(i_{1}, i_{2}\right),\left(j_{1}, j_{2}\right)$, if the terms involving $\left(\alpha_{i_{1}} \alpha_{i_{2}}\right)^{M_{2}}$ and $\left(\alpha_{j_{1}} \alpha_{j_{2}}\right)^{M_{2}}$ occur in the same class of $\mathcal{P}$, then

$$
\left(\alpha_{i_{1}} \alpha_{i_{2}}\right)^{z_{1}}=\left(\alpha_{j_{1}} \alpha_{j_{2}}\right)^{z_{1}}
$$

We again use Lemma 3 with $S_{1}$ to get a contradiction in this case. Put $p=q=z_{1}$, and observe that by our assumptions

$$
m_{1} \neq m_{1}^{\prime} \text { and } m_{2} \neq m_{2}^{\prime},
$$

as $z_{1}$ is the difference of two distinct solutions $M_{2}$ and $M_{2}^{\prime}$ of (8), we may suppose that

$$
p \neq 0 \quad \text { and } \quad q \neq 0 \text {. }
$$

We apply Lemma 3 to the partition $\mathcal{P}=\left\{T_{1}, \ldots, T_{t}\right\}$ corresponding to our vanishing subsums in (8). Note that by $p=q$ we now have $|\Pi|=10$ in Lemma 3. Just as in part 1) of the proof, we see that the ratio of two of the above $\alpha_{i}$ 's is a root of unity. This contradiction yields that (a) must hold also in case 2).

Suppose now that (a) is valid but (b) is not. Hence some subgraph $V_{L}$ of $V$ is disconnected. By an analysis of the possibilities we see that without loss of generality we may assume that

$$
V_{L} \subseteq\left\{\left\{\alpha_{1}, \alpha_{2}, \alpha_{3}\right\},\left\{\alpha_{1}, \alpha_{2}, \alpha_{4}\right\},\left\{\alpha_{1}, \alpha_{2}, \alpha_{5}\right\},\left\{\alpha_{3}, \alpha_{4}, \alpha_{5}\right\}\right\} .
$$

Thus for the set $I$ of the index-triplets of the vanishing determinants $D_{i_{3} i_{4} i_{5}}$ we obtain

$$
I \subseteq\{\{1,2,3\},\{1,2,4\},\{1,2,5\},\{3,4,5\}\} .
$$

Now we can repeat the whole argument above, and by the help of Lemmas 1 and 3 with $S=S_{2}$, we get a contradiction also in this case.

Suppose now that (a) and (b) are valid but $\left(c^{\prime}\right)$ is not, i.e. some subgraph $V_{L}$ of $V$ is of that special form. Without loss of generality we may assume that

$$
V_{L} \subseteq\left\{\left\{\alpha_{1}, \alpha_{2}, \alpha_{3}\right\},\left\{\alpha_{1}, \alpha_{2}, \alpha_{4}\right\},\left\{\alpha_{1}, \alpha_{3}, \alpha_{5}\right\}\right\}
$$


Thus for the set $I$ of the index-triplets of the vanishing determinants $D_{i_{3} i_{4} i_{5}}$ we obtain

$$
I \subseteq\{\{1,2,3\},\{1,2,4\},\{1,3,5\}\} .
$$

We can repeat the whole process again, this time with case $S_{3}$ of Lemma 3 , and arrive at a contradiction. The proof of the lemma is now complete in the case of (i).

Suppose now that (ii) holds. Choose any $Q \in A$ with exponent quintuple $\left(m_{1}, m_{2}, m_{3}, m_{4}, 0\right)$ and put $\left(M_{2}, M_{3}, M_{4}\right)=\left(m_{2}, m_{3}, m_{4}\right)$. The fact that $P$ divides all the standard quintinomials in $A$ means that the equation

$$
\left|\begin{array}{ccccc}
\alpha_{1}^{M_{1}} & \alpha_{2}^{M_{1}} & \alpha_{3}^{M_{1}} & \alpha_{4}^{M_{1}} & \alpha_{5}^{M_{1}} \\
\alpha_{1}^{M_{2}+M} & \alpha_{2}^{M_{2}+M} & \alpha_{3}^{M_{2}+M} & \alpha_{4}^{M_{2}+M} & \alpha_{5}^{M_{2}+M} \\
\alpha_{1}^{M_{3}+M} & \alpha_{2}^{M_{3}+M} & \alpha_{3}^{M_{3}+M} & \alpha_{4}^{M_{3}+M} & \alpha_{5}^{M_{3}+M} \\
\alpha_{1}^{M_{4}+M} & \alpha_{2}^{M_{4}+M} & \alpha_{3}^{M_{4}+M} & \alpha_{4}^{M_{4}+M} & \alpha_{5}^{M_{4}+M} \\
1 & 1 & 1 & 1 & 1
\end{array}\right|=0
$$

for any fixed set $\left\{\alpha_{1}, \alpha_{2}, \alpha_{3}, \alpha_{4}, \alpha_{5}\right\}$ of pairwise non-equivalent roots of $P$ has infinitely many solutions in integers $M_{1}, M$ with $M_{1}>M_{2}$. If we put

$$
D_{i_{1} i_{2} i_{3}}=\left|\begin{array}{ccc}
\alpha_{i_{1}}^{M_{2}} & \alpha_{i_{2}}^{M_{2}} & \alpha_{i_{3}}^{M_{2}} \\
\alpha_{i_{1}}^{M_{3}} & \alpha_{i_{2}}^{M_{3}} & \alpha_{i_{3}}^{M_{3}} \\
\alpha_{i_{1}}^{M_{4}} & \alpha_{i_{2}}^{M_{4}} & \alpha_{i_{3}}^{M_{4}}
\end{array}\right|
$$

for all $\left\{i_{1}, i_{2}, i_{3}\right\}$ with $1 \leq i_{1}<i_{2}<i_{3} \leq 5$, the previous equation can be rewritten as

$$
\sum_{\substack{\left\{i_{1}, i_{2}, i_{3}, i_{4}, i_{5}\right\}=\{1,2,3,4,5\} \\ i_{3}<i_{4}<i_{5}}}(-1)^{i_{1}+i_{2}} \operatorname{sgn}\left(i_{2}-i_{1}\right) D_{i_{3} i_{4} i_{5}} \alpha_{i_{1}}^{M_{1}}\left(\alpha_{i_{3}} \alpha_{i_{4}} \alpha_{i_{5}}\right)^{M}=0,
$$

hence after dividing by $\left(\alpha_{1} \alpha_{2} \alpha_{3} \alpha_{4} \alpha_{5}\right)^{M}$ and omitting the terms with $D_{i_{3} i_{4} i_{5}}$ $=0$, as

$$
\sum_{\substack{\left\{i_{1}, i_{2}, i_{3}, i_{4}, i_{5}\right\}=\{1,2,3,4,5\} \\ i_{3}<i_{4}<i_{5}}}^{*}(-1)^{i_{1}+i_{2}+1} \operatorname{sgn}\left(i_{2}-i_{1}\right) D_{i_{3} i_{4} i_{5}} \alpha_{i_{1}}^{M_{1}-M} \alpha_{i_{2}}^{-M}=0 .
$$

If $Q_{1}, Q_{2}$ are any two polynomials in $A$ with exponent quintuples $\left(M_{1}\right.$, $\left.M_{2}+M, M_{3}+M, M_{4}+M, 0\right)$ and $\left(M_{1}^{\prime}, M_{2}+M^{\prime}, M_{3}+M^{\prime}, M_{4}+M^{\prime}, 0\right)$, respectively, then any of the equalities

$$
\begin{gathered}
M_{1}-M=M_{1}^{\prime}-M^{\prime}, \quad-M=-M^{\prime}, \\
\left(M_{1}-M\right)-\left(M_{1}^{\prime}-M^{\prime}\right)= \pm\left(-M-\left(-M^{\prime}\right)\right)
\end{gathered}
$$

would contradict our assumptions concerning these exponent quintuples. Now we can apply the whole process used to prove the lemma in case 1) of 
(i) to (9). By the help of Lemmas 1 and 3, our result also follows in this case. Note that by the impossibility of the previous equations we have

$$
p \neq 0, \quad q \neq 0, \quad p \neq \pm q,
$$

whence $|\Pi|=20$ in Lemma 3 in this case.

It will be convenient to deal with a special case of Theorem 2 in the following lemma separately.

Lemma 5. Using the notation of Theorem 2, let $A$ be an infinite set of standard quintinomials divisible by $P$ over $\mathbb{Q}$. Suppose that all the polynomials in $A$ have exponent quintuples of the form $\left(m_{1}, M_{2}, M_{3}, M_{4}, 0\right)$ with the same fixed $M_{2}, M_{3}, M_{4}$. Then either condition (i) or (ii) of Theorem 2 is valid for $P$.

Proof. Write $X=m_{1}$, and observe that as $P$ divides the standard quintinomials in $A$ over $\mathbb{Q}$, the equation

$$
\left|\begin{array}{ccccc}
\alpha_{1}^{X} & \alpha_{2}^{X} & \alpha_{3}^{X} & \alpha_{4}^{X} & \alpha_{5}^{X} \\
\alpha_{1}^{M_{2}} & \alpha_{2}^{M_{2}} & \alpha_{3}^{M_{2}} & \alpha_{4}^{M_{2}} & \alpha_{5}^{M_{2}} \\
\alpha_{1}^{M_{3}} & \alpha_{2}^{M_{3}} & \alpha_{3}^{M_{3}} & \alpha_{4}^{M_{3}} & \alpha_{5}^{M_{3}} \\
\alpha_{1}^{M_{4}} & \alpha_{2}^{M_{4}} & \alpha_{3}^{M_{4}} & \alpha_{4}^{M_{4}} & \alpha_{5}^{M_{4}} \\
1 & 1 & 1 & 1 & 1
\end{array}\right|=0
$$

has infinitely many solutions in integers $X$ with $X>M_{2}$. If we put

$$
D_{i_{1} i_{2} i_{3} i_{4}}=\left|\begin{array}{cccc}
\alpha_{i_{1}}^{M_{2}} & \alpha_{i_{2}}^{M_{2}} & \alpha_{i_{3}}^{M_{2}} & \alpha_{i_{4}}^{M_{2}} \\
\alpha_{i_{1}}^{M_{3}} & \alpha_{i_{2}}^{M_{3}} & \alpha_{i_{3}}^{M_{3}} & \alpha_{i_{3}}^{M_{3}} \\
\alpha_{i_{1}}^{M_{4}} & \alpha_{i_{2}}^{M_{4}} & \alpha_{i_{3}}^{M_{4}} & \alpha_{i_{4}}^{M_{4}} \\
1 & 1 & 1 & 1
\end{array}\right|
$$

for all $\left\{i_{1}, i_{2}, i_{3}, i_{4}\right\}$ with $1 \leq i_{1}<i_{2}<i_{3}<i_{4} \leq 5$, the previous equation can be rewritten as

$$
D_{2345} \alpha_{1}^{X}-D_{1345} \alpha_{2}^{X}+D_{1245} \alpha_{3}^{X}-D_{1235} \alpha_{4}^{X}+D_{1234} \alpha_{5}^{X}=0 .
$$

Suppose that in (10) at least one of the subdeterminants $D_{i_{1} i_{2} i_{3} i_{4}}$ does not vanish. Note that as the $\alpha_{i}$ 's are non-zero, at least two such determinants do not vanish in this case. Choose a partition $\mathcal{P}$ of the left hand side of (10) into subsums which vanish for infinitely many $X$ (belonging to the $Q$ 's in $A$ ), but have proper vanishing subsums only in finitely many instances. Omit all the $Q$ 's from $A$ for which these subsums either do not vanish, or have proper vanishing subsums. Observe that we still have $|A|=\infty$. Applying Lemma 1 to the partition $\mathcal{P}$, we immediately obtain $G(\mathcal{P}) \neq\{\underline{0}\}$. Hence for 
some non-zero integer $z_{1}$ and indices $i_{1}, i_{2}$ with $1 \leq i_{1}<i_{2} \leq 5$ we have

$$
\alpha_{i_{1}}^{z_{1}}=\alpha_{i_{2}}^{z_{1}},
$$

which contradicts the non-equivalence of $\alpha_{i_{1}}$ and $\alpha_{i_{2}}$. This shows that all the determinants $D_{i_{1} i_{2} i_{3} i_{4}}$ must vanish in (10).

Hence for some polynomials

$$
F_{j}(x)=x^{M_{2}}+a_{j} x^{M_{3}}+b_{j} x^{M_{4}}+c_{j} \quad(j=1,2,3,4,5)
$$

with $a_{j}, b_{j}, c_{j} \in \mathbb{K}$ and $\left(a_{j}, b_{j}, c_{j}\right) \neq(0,0,0)$ we have

$$
F_{j}\left(\alpha_{i}\right)=0 \quad \text { for } i, j \in\{1,2,3,4,5\}, i \neq j .
$$

Observe that if for some $j$ and $j^{\prime}$ we have $F_{j} \neq F_{j^{\prime}}$, then there exist three $\alpha_{i}$ 's such that they are roots of the distinct standard quadrinomials $F_{j}$ and $F_{j^{\prime}}$, both having exponent quadruple $\left(M_{2}, M_{3}, M_{4}, 0\right)$. Without loss of generality we may assume that these $\alpha_{i}$ 's are $\alpha_{1}, \alpha_{2}, \alpha_{3}$ and $j=4, j^{\prime}=5$. Further, we may assume that $a_{4} \neq a_{5}$, otherwise $\alpha_{1}, \alpha_{2}, \alpha_{3}$ would be roots of the same binomial $F_{4}-F_{5}$, whence all their ratios would be roots of unity. So $\alpha_{1}, \alpha_{2}, \alpha_{3}$ are also roots of the standard trinomial

$$
F_{6}(x):=\frac{1}{a_{4}-a_{5}}\left(F_{4}(x)-F_{5}(x)\right) .
$$

Recalling that, for infinitely many positive integers $m_{1}, P$ divides some standard quintinomial $Q_{m_{1}}(x)$ having exponent quintuple $\left(m_{1}, M_{2}, M_{3}, M_{4}, 0\right)$, we find that the polynomial

$$
P_{0}(x)=\left(x-\alpha_{1}\right)\left(x-\alpha_{2}\right)\left(x-\alpha_{3}\right)
$$

divides over $\mathbb{K}$ infinitely many standard trinomials of the form

$$
Q_{m_{1}}(x)-C_{1} F_{4}(x)-C_{2} F_{6}(x)
$$

where $C_{1}$ and $C_{2}$ are appropriately chosen numbers from $\mathbb{K}$. Now by Theorem 1 of [2], for some positive integer $r$ and some polynomial $R \in \mathbb{K}[x]$ of degree at most two, $P_{0}$ divides $R\left(x^{r}\right)$ over $\mathbb{K}$. (We note that this assertion is proved only for $\mathbb{K}=\mathbb{Q}$ in [2], but as mentioned by the authors, this more general statement is also valid.) Hence for some $1 \leq i_{1}<i_{2} \leq 3$ the ratio $\alpha_{i_{1}} / \alpha_{i_{2}}$ is a root of unity. However, this contradicts the non-equivalence of these roots. Thus we obtain

$$
F_{j}=F_{j^{\prime}}, \quad j, j^{\prime} \in\{1,2,3,4,5\} .
$$

However, if we take any other system of non-equivalent $\alpha_{i}$ 's, the whole argument given above remains valid. Hence, as $P$ has only simple roots, we may conclude that $P \mid F_{1}$ over $\mathbb{K}$.

Suppose now that $\sigma\left(F_{1}\right) \neq F_{1}$ for some automorphism $\sigma$ of $\mathbb{K}$. As clearly $\sigma(P)=P$, this implies $P \mid \sigma\left(F_{1}\right)$, whence

$$
P(x) \mid\left(F_{1}(x)-\sigma\left(F_{1}(x)\right)\right)=: c_{0} F_{0}(x)
$$


over $\mathbb{K}$, where $c_{0}$ is the leading coefficient of the polynomial $F_{1}-\sigma\left(F_{1}\right)$. Observe that $F_{0}(x)$ is a standard trinomial with exponent triple $\left(M_{3}, M_{4}, 0\right)$ and with coefficients from $\mathbb{K}$. Thus if $F_{0} \notin \mathbb{Q}[x]$, then repeating the previous argument with $F_{0}$ in place of $F_{1}$, we deduce that $P$ divides a binomial over $\mathbb{K}$. This would yield that the ratio of each pair of roots of $P$ is a root of unity, which is a contradiction. Hence we may assume that either $\sigma\left(F_{1}\right)=F_{1}$ for all the automorphisms of $\mathbb{K}$, whence $F_{1} \in \mathbb{Q}[x]$, or $F_{0}(x) \in \mathbb{Q}[x]$. Hence we have

$$
P \mid F_{1} \quad \text { or } \quad P \mid F_{0}
$$

over $\mathbb{Q}$. In the first case put $G=F_{1}$, otherwise let $G=F_{0}$. By the fact that for any $Q \in A$ the polynomial $Q+G$ is a standard quintinomial having the same exponent quintuple as $Q$, and clearly $P \mid Q+G$, the lemma follows.

Proof of Theorem 2. The "if" part of the theorem can be proved by the same argument as in the case of Theorem 1.

To prove the "only if" part, suppose that $P$ divides infinitely many standard quintinomials with non-zero constant terms over $\mathbb{Q}$. Let $A$ be an infinite set of such standard quintinomials. If there are infinitely many standard quadrinomials in $A$, then our statement is an immediate consequence of Theorem 1. Hence we may assume that there are no standard quadrinomials in $A$. Moreover, we may suppose that the exponent quintuples of the standard quintinomials in $A$ are all distinct, otherwise the theorem follows. Finally, we may also assume that one of the three statements

$$
\begin{aligned}
\left(m_{3}, m_{4}\right) & =\left(m_{3}^{\prime}, m_{4}^{\prime}\right) \quad \text { for all } Q_{1}, Q_{2} \in A, \\
\left(m_{2}-m_{4}, m_{3}-m_{4}\right) & =\left(m_{2}^{\prime}-m_{4}^{\prime}, m_{3}^{\prime}-m_{4}^{\prime}\right) \quad \text { for all } Q_{1}, Q_{2} \in A, \\
\left(m_{1}-m_{3}, m_{2}-m_{3}\right) & =\left(m_{1}^{\prime}-m_{3}^{\prime}, m_{2}^{\prime}-m_{3}^{\prime}\right) \quad \text { for all } Q_{1}, Q_{2} \in A
\end{aligned}
$$

holds, where $\left(m_{1}, m_{2}, m_{3}, m_{4}, 0\right)$ and $\left(m_{1}^{\prime}, m_{2}^{\prime}, m_{3}^{\prime}, m_{4}^{\prime}, 0\right)$ are the exponent quintuples of $Q_{1}$ and $Q_{2}$, respectively. Indeed, otherwise by a similar argument as in the case of quadrinomials, using Lemma 2 we would get $P \in \mathrm{PR}_{5}$, which would prove the theorem. Denote the splitting field of the polynomial $P$ by $\mathbb{K}$.

(A) Assume that for any $Q_{1}, Q_{2} \in A$ with exponent quintuples $\left(m_{1}, m_{2}\right.$, $\left.m_{3}, m_{4}, 0\right)$ and $\left(m_{1}^{\prime}, m_{2}^{\prime}, m_{3}^{\prime}, m_{4}^{\prime}, 0\right)$, respectively, we have

$$
\left(m_{3}, m_{4}\right)=\left(m_{3}^{\prime}, m_{4}^{\prime}\right)
$$

Put $\left(M_{3}, M_{4}\right)=\left(m_{3}, m_{4}\right)$. We may assume that the roots of $P$ belong to at least five equivalence classes, otherwise we immediately have $P \in \mathrm{PR}_{5}$.

We will use Lemma 4 to derive the theorem in this case. Suppose first that there are infinitely many polynomials in $A$ with the same exponent $m_{1}$ or the same exponent $m_{2}$. 
In the former case $P$ divides infinitely many standard quintinomials of the same degree, hence divides two such polynomials with the same exponent quintuple.

In the latter case $P$ divides infinitely many standard quintinomials having exponent quintuples of the form $\left(m_{1}, M_{2}, M_{3}, M_{4}, 0\right)$, with the same fixed $M_{2}, M_{3}, M_{4}$. Hence Lemma 5 implies the theorem in this case.

So there is an infinite subset of $A$, which we will still call $A$ in what follows, such that for all the polynomials $Q_{1}, Q_{2} \in A$ with exponent quintuples $\left(m_{1}, m_{2}, m_{3}, m_{4}, 0\right)$ and $\left(m_{1}^{\prime}, m_{2}^{\prime}, m_{3}^{\prime}, m_{4}^{\prime}, 0\right)$, respectively, we have

$$
m_{1} \neq m_{1}^{\prime} \quad \text { and } \quad m_{2} \neq m_{2}^{\prime} \text {. }
$$

Observe that now all the assumptions of Lemma 4(i) are satisfied. We note that the determinant

$$
\left|\begin{array}{ccc}
\alpha_{i_{1}}^{M_{3}} & \alpha_{i_{2}}^{M_{3}} & \alpha_{i_{3}}^{M_{3}} \\
\alpha_{i_{1}}^{M_{4}} & \alpha_{i_{2}}^{M_{4}} & \alpha_{i_{3}}^{M_{4}} \\
1 & 1 & 1
\end{array}\right|
$$

vanishes if and only if $\alpha_{i_{1}}, \alpha_{i_{2}}, \alpha_{i_{3}}$ are the roots of some standard trinomial having coefficients from $\mathbb{K}$, and with exponent triple $\left(M_{3}, M_{4}, 0\right)$. Since the three corresponding roots of $P$ are pairwise non-equivalent, none of these trinomials is a binomial. We claim that all these standard trinomials coincide. Suppose to the contrary that for some triplets $\left\{\alpha_{i_{1}}, \alpha_{i_{2}}, \alpha_{i_{3}}\right\}$ and $\left\{\alpha_{j_{1}}, \alpha_{j_{2}}, \alpha_{j_{3}}\right\}$ consisting of pairwise non-equivalent roots of $P$, the corresponding standard trinomials are different. By Lemma 4 we deduce that the corresponding graph $V$ is connected, whence there exist two triplets $\left\{\alpha_{i_{1}}^{\prime}, \alpha_{i_{2}}^{\prime}, \alpha_{i_{3}}^{\prime}\right\}$ and $\left\{\alpha_{j_{1}}^{\prime}, \alpha_{j_{2}}^{\prime}, \alpha_{j_{3}}^{\prime}\right\}$ such that they are neighbours in $V$, and the standard trinomials corresponding to them, say $F_{1}$ and $F_{2}$, respectively, are different. However, then by the definition of $V$,

$$
\left|\left\{\alpha_{i_{1}}^{\prime}, \alpha_{i_{2}}^{\prime}, \alpha_{i_{3}}^{\prime}\right\} \cap\left\{\alpha_{j_{1}}^{\prime}, \alpha_{j_{2}}^{\prime}, \alpha_{j_{3}}^{\prime}\right\}\right|=2 .
$$

Thus $P$ has two non-equivalent roots, which are also roots of the binomial $F_{1}-F_{2}$, but this is a contradiction. It shows that all these standard trinomials coincide. As every root of $P$ is contained in some vertex of $V$ and $P$ has only simple roots, we see that $P$ divides a standard trinomial $F \in \mathbb{K}[x]$ over $\mathbb{K}$. Suppose that the coefficients of $F$ are not rational. Then there exists an automorphism $\sigma$ of $\mathbb{K}$ such that $\sigma(F) \neq F$. This together with $\sigma(P)=P$ yields $P \mid(F-\sigma(F))$, whence the roots of $P$ are roots of a binomial. However, this contradicts our assumption that the roots of $P$ belong to at least five equivalence classes. Thus $F \in \mathbb{Q}[x]$. Finally, observe that any standard trinomial having rational coefficients divides over $\mathbb{Q}$ infinitely many standard quintinomials with the same exponent quintuple. In fact the trinomial $x^{n_{1}}+a_{1} x^{n_{2}}+a_{2}$ divides the quintinomial $\left(x^{n_{1}}+a_{1} x^{n_{2}}+a_{2}\right)\left(x^{n_{2}}+b\right)$ for every $b \in \mathbb{Q}$. Thus the proof of the theorem is complete in case $(\mathrm{A})$. 
(B) Omitting all the other polynomials, now suppose that for any $Q_{1}, Q_{2}$ with exponent quintuples $\left(m_{1}, m_{2}, m_{3}, m_{4}, 0\right)$ and $\left(m_{1}^{\prime}, m_{2}^{\prime}, m_{3}^{\prime}, m_{4}^{\prime}, 0\right)$, respectively, in the infinite set $A$ we have

$$
\left(m_{2}-m_{4}, m_{3}-m_{4}\right)=\left(m_{2}^{\prime}-m_{4}^{\prime}, m_{3}^{\prime}-m_{4}^{\prime}\right) .
$$

Observe that under the assumption

$$
m_{1} \neq m_{1}^{\prime}, \quad m_{2} \neq m_{2}^{\prime} \quad \text { and } \quad \frac{m_{1}-m_{2}}{m_{1}^{\prime}-m_{2}^{\prime}} \notin\{1,2\}
$$

we can use Lemma 4 again, and by repeating the argument given in case (A), the theorem follows also in this case (B). So assume that in the case of any $Q_{1}$ and $Q_{2}$ as above, we also have

$$
m_{1}=m_{1}^{\prime}, \quad m_{2}=m_{2}^{\prime} \quad \text { or } \quad \frac{m_{1}-m_{1}^{\prime}}{m_{2}-m_{2}^{\prime}} \in\{1,2\} .
$$

By applying the box principle we may assume that all elements of $A$ satisfy the same relation.

In the case of $m_{1}=m_{1}^{\prime}$ we deduce that $P$ divides infinitely many standard trinomials of the same degree, whence $P$ divides two such polynomials having the same exponent quintuple, and the theorem follows.

If $m_{2}=m_{2}^{\prime}$, then also $m_{3}=m_{3}^{\prime}$ and $m_{4}=m_{4}^{\prime}$, and by Lemma 5 we are done.

If $m_{1}-m_{1}^{\prime}=2\left(m_{2}-m_{2}^{\prime}\right)$, then for some integers $M_{1}, M_{2}, M_{3}, M_{4}$ the exponent quintuples of $Q_{1}$ and $Q_{2}$ are of the forms

$$
\left(M_{1}+2 M, M_{2}+M, M_{3}+M, M_{4}+M, 0\right)
$$

and

$$
\left(M_{1}+2 M^{\prime}, M_{2}+M^{\prime}, M_{3}+M^{\prime}, M_{4}+M^{\prime}, 0\right)
$$

with some integers $M$ and $M^{\prime}$, respectively. This implies condition (iii), and the theorem follows.

Suppose that

$$
m_{1}-m_{2}=m_{1}^{\prime}-m_{2}^{\prime}
$$

This means that also $m_{1}-m_{3}=m_{1}^{\prime}-m_{3}^{\prime}$ and $m_{1}-m_{4}=m_{1}^{\prime}-m_{4}^{\prime}$, whence $P$ divides over $\mathbb{Q}$ infinitely many standard trinomials of the form

$$
Q_{M}(x)=x^{M_{1}+M}+a_{M} x^{M_{2}+M}+b_{M} x^{M_{3}+M}+c_{M} x^{M_{4}+M}+d_{M},
$$

where $d_{M} \neq 0, M$ is a positive integer and $M_{1}, M_{2}, M_{3}, M_{4}$ are fixed integers with $M_{1}>M_{2}>M_{3}>M_{4}>0$. Put $P^{*}(x)=x^{\operatorname{deg}(P)} P(1 / x)$. Observe that $P^{*}$ divides all the standard quintinomials

$$
\begin{aligned}
x^{M_{1}+M}+\left(c_{M} / d_{M}\right) & x^{M_{1}-M_{4}} \\
& +\left(b_{M} / d_{M}\right) x^{M_{1}-M_{3}}+\left(a_{M} / d_{M}\right) x^{M_{1}-M_{2}}+1 / d_{M}
\end{aligned}
$$


over $\mathbb{Q}$. However, for such polynomials $P^{*}$ we already proved the statement in Lemma 5, so we infer that either $P^{*} \in \mathrm{PR}_{5}$, or $P^{*}$ divides two quintinomials with the same exponent quintuple. Now just as at the end of the proof of Theorem 1, we conclude that the same is true also for $P$, which verifies our statement in case (B).

(C) Finally suppose that after omitting all the other polynomials, for any $Q_{1}, Q_{2}$ with exponent quintuples $\left(m_{1}, m_{2}, m_{3}, m_{4}, 0\right)$ and $\left(m_{1}^{\prime}, m_{2}^{\prime}, m_{3}^{\prime}\right.$, $\left.m_{4}^{\prime}, 0\right)$, respectively, in some infinite set $A$ we have

$$
\left(m_{1}-m_{3}, m_{2}-m_{3}\right)=\left(m_{1}^{\prime}-m_{3}^{\prime}, m_{2}^{\prime}-m_{3}^{\prime}\right) .
$$

Put $P^{*}=x^{\operatorname{deg}(P)} P(1 / x)$ and write the polynomials $Q_{i} \in A(i \in \mathbb{N})$ in the form

$$
Q_{i}(x)=x^{m_{1}^{(i)}}+a_{i} x^{m_{2}^{(i)}}+b_{i} x^{m_{3}^{(i)}}+c_{i} x^{m_{4}^{(i)}}+d_{i} .
$$

Note that $d_{i} \neq 0$ for all $Q_{i}$, and put $P^{*}(x)=x^{\operatorname{deg}(P)} P(1 / x)$. Observe that $P^{*}$ divides all the standard quintinomials

$$
x^{m_{1}^{(i)}}+\left(c_{i} / d_{i}\right) x^{m_{1}^{(i)}-m_{4}^{(i)}}+\left(b_{i} / d_{i}\right) x^{m_{1}^{(i)}-m_{3}^{(i)}}+\left(a_{i} / d_{i}\right) x^{m_{1}^{(i)}-m_{2}^{(i)}}+1 / d_{i}
$$

over $\mathbb{Q}$. However, for such polynomials $P^{*}$ we already proved the theorem in part (A). So we infer that either $P^{*} \in \mathrm{PR}_{5}$, or $P^{*}$ divides two quintinomials with the same exponent quintuple. Just as at the end of the proof of Theorem 1, we conclude that the same is true also for $P$, which verifies our statement in case $(\mathrm{C})$.

\section{The necessity of condition (ii)}

Proof of the Proposition. Let $P(x)=x^{5}-8 x^{2}-16 x+16$. Observe that the degree of $P$ is prime, that $P$ is irreducible over $\mathbb{Q}$ and that $P$ has two roots of different absolute values. By Lemma 2 of [3] these properties imply that $P \notin \mathrm{PR}_{5}$.

To prove that $P$ does not divide any trinomial over $\mathbb{Q}$, consider the roots

$$
\begin{gathered}
\alpha_{1}=-0.6128676243 \ldots+i \cdot 2.209220116 \ldots, \\
\alpha_{2}=-1.799759775 \ldots, \quad \alpha_{3}=0.7400417854 \ldots
\end{gathered}
$$

of $P$. Suppose that $P$ divides a standard trinomial over $\mathbb{Q}$ having exponent triple $\left(m_{1}, m_{2}, 0\right)$. Then $m_{1} \geq 6$ and the determinant

$$
\left|\begin{array}{ccc}
\alpha_{1}^{m_{1}} & \alpha_{2}^{m_{1}} & \alpha_{3}^{m_{1}} \\
\alpha_{1}^{m_{2}} & \alpha_{2}^{m_{2}} & \alpha_{3}^{m_{2}} \\
1 & 1 & 1
\end{array}\right|
$$

must vanish. After dividing by $\alpha_{1}^{m_{1}} \alpha_{2}^{m_{2}}$, we obtain 


$$
\begin{aligned}
1+\left(\frac{\alpha_{2}}{\alpha_{1}}\right)^{m_{1}}\left(\frac{\alpha_{3}}{\alpha_{2}}\right)^{m_{2}}+ & \left(\frac{\alpha_{2}}{\alpha_{1}}\right)^{m_{1}-m_{2}}\left(\frac{\alpha_{3}}{\alpha_{2}}\right)^{m_{1}} \\
& -\left(\frac{\alpha_{3}}{\alpha_{1}}\right)^{m_{1}}-\left(\frac{\alpha_{3}}{\alpha_{2}}\right)^{m_{2}}-\left(\frac{\alpha_{2}}{\alpha_{1}}\right)^{m_{1}-m_{2}}=0 .
\end{aligned}
$$

However, if we observe that $\max \left(m_{2}, m_{1}-m_{2}\right) \geq 3$, a simple calculation shows that

$1-\left|\frac{\alpha_{2}}{\alpha_{1}}\right|^{m_{1}}\left|\frac{\alpha_{3}}{\alpha_{2}}\right|^{m_{2}}-\left|\frac{\alpha_{2}}{\alpha_{1}}\right|^{m_{1}-m_{2}}\left|\frac{\alpha_{3}}{\alpha_{2}}\right|^{m_{1}}-\left|\frac{\alpha_{3}}{\alpha_{1}}\right|^{m_{1}}-\left|\frac{\alpha_{3}}{\alpha_{2}}\right|^{m_{2}}-\left|\frac{\alpha_{2}}{\alpha_{1}}\right|^{m_{1}-m_{2}}>0$.

This contradiction yields that $P$ cannot divide any standard trinomial over $\mathbb{Q}$.

Finally, one can readily verify that $P$ divides $Q=x^{12}-3328 x^{2}-3072 x+$ 4096, hence $Q+a P$ for all $a \in \mathbb{Q}$.

Acknowledgements. The first author is grateful to Leiden University for its hospitality during this research. We thank the referee for his valuable remarks.

\section{References}

[1] J.-H. Evertse, H. P. Schlickewei and W. M. Schmidt, Linear equations in variables which lie in a multiplicative group, Ann. of Math. 155 (2002), 1-30.

[2] K. Györy and A. Schinzel, On a conjecture of Posner and Rumsey, J. Number Theory 47 (1994), 63-78.

[3] L. Hajdu, On a problem of Györy and Schinzel concerning polynomials, Acta Arith. 78 (1997), 287-295.

[4] E. C. Posner and H. Rumsey, Jr., Polynomials that divide infinitely many trinomials, Michigan Math. J. 12 (1965), 339-348.

[5] H. P. Schlickewei and C. Viola, Polynomials that divide many trinomials, Acta Arith. 78 (1997), 267-273.

[6] - - - Polynomials that divide many k-nomials, in: Number Theory in Progress (Zakopane, 1997), K. Győry, H. Iwaniec and J. Urbanowicz (eds.), Vol. 1, de Gruyter, Berlin, 1999, 445-450.

Institute of Mathematics and Informatics

Mathematical Institute University of Debrecen

Leiden University P.O. Box 9512

P.O. Box 12

4010 Debrecen, Hungary

E-mail: hajdul@math.klte.hu

2300 RA Leiden, The Netherlands

E-mail: tijdeman@math.leidenuniv.nl

Received on 18.2.2002

and in revised form on 29.7.2002 